\title{
The pineal hormone melatonin attenuates doxorubicin induced-mitochondrial dysfunction and cardiotoxicity in vitro
}

\author{
Thulangana K Ganeshan, Jimmy D Bell, Nelson W Chong \\ Department of Life Sciences, University of Westminster, London, UK
}

Background: Heart failure (HF) is a major end-point of cardiovascular diseases (CVD). The pathogenesis of HF is mostly unresolved but involves metabolic alterations. Treatment of animals and cardiomyocytes with $\beta$-adrenergic receptor agonists induces HF. Mitochondrial dysfunction and HF are common complications of anticancer drugs such as doxorubicin (DOX). Melatonin synthesis dramatically decreases with age and in patients with CVD. The aim of this study was to investigate whether DOX-induced cardiac dysfunction can be attenuated by melatonin.

Methods: The Seahorse XF analyser was utilised (with the XFp Cell Energy Phenotype kit) to measure oxygen consumption rate [OCR; oxidative phosphorylation (OXPHOS)] and extracellular acidification rate (ECAR; glycolysis) in living rat cardiomyocyte-derived $\mathrm{H} 9 \mathrm{c} 2$ cell line. Mono-layers of cells were treated with cardiotoxic drugs [isoproterenol (ISO, $100 \mu \mathrm{M})$ or DOX $(0.1 \mu \mathrm{M})$ ] for $24 \mathrm{hr}$ with and without melatonin co-treatment (MEL, 1 $\mu \mathrm{M})$. Cyan ADP flow cytometry was used to examine the anti-apoptotic properties of MEL $(1 \mu \mathrm{M})$ on DOX-treatment $(0.5 \mu \mathrm{M}, 24 \mathrm{hr})$. Data are given as mean \pm SEM ( $n=$ separate experiments) and analysis was performed using ANOVA and two-tail unpaired Student's T-test, as applicable.

Results: Isoproterenol-treatment increased peak OCR of H9c2 cells by 30\% which was inhibited by MEL [CON, 384 \pm 17 ; ISO, $496 \pm 33$; ISO+MEL, $412 \pm 31 \mathrm{pmol} / \mathrm{min}$; $\mathrm{n}=3$ (six replicates); CON vs. ISO, $\mathrm{p}<0.05$; ISO vs. ISO+MEL, $\mathrm{p}<0.05$; CON vs. ISO+MEL, $\mathrm{p}>0.05]$. Doxorubicin-treatment decreased OCR by $\sim 40 \%$ which was reversed by MEL [CON, $934 \pm 69$; DOX, 554 \pm 52; DOX+MEL, $858 \pm 97 \mathrm{pmol} / \mathrm{min} ; \mathrm{n}=3$ (six replicates); CON vs. DOX, p<0.05; DOX vs. DOX + MEL, $p<0.05$; CON vs. DOX + MEL, $>0.05$ ]. ISO and DOX significantly increased $(\sim 30 \%)$ and decreased $(\sim$ 25\%) ECAR respectively $(n=3, p<0.05)$ which was not inhibited by MEL. Melatonin alone had no significant effect on OCR and ECAR. Melatonin inhibited DOX-induced apoptosis in H9c2 cells [CON, $6.3 \pm 0.8 \%$; DOX, $22 \pm 1.8 \%$; DOX + MEL, $11 \pm 1.7 \%, n=4$ (two replicates); CON vs. DOX, $p<0.001$; DOX vs. DOX+MEL, $p<0.004$; CON vs. DOX+MEL, $\mathrm{p}>0.05]$.

Conclusions: ISO and DOX-treatment induced mitochondrial dysfunction in H9c2 cells by alteration of OXPHOS and glycolysis; changes in OXPHOS were prevented by MEL. These data indicate that DOX-induced apoptosis in cardiac cells may be mediated, at least in part, by OXPHOS dysfunction which was attenuated by MEL treatment. 